\section{PWE-200 MR ENTEROGRAPHY IS A USEFUL TEST IN THE INVESTIGATION OF SMALL BOWEL DISEASE}

doi:10.1136/gutjnl-2013-304907.488

1."S Soteriadou, ' $\mathrm{K}$ White, ${ }^{2} \mathrm{R}$ Filobbos, ${ }^{1} \mathrm{~J} \mathrm{~K}$ Limdi. ' $\mathrm{G}$ Gastroenterology; ${ }^{2}$ Radiology, Pennine Acute NHS Trust, Manchester, UK

Introduction $\mathrm{MR}$ enterography (MRE) aids assessment of small bowel (SB) inflammatory bowel disease (IBD). We aimed to determine the frequency and clinical impact of incidental findings detected by MRE in patients with suspected or known Crohn's disease (CD).

Methods We conducted a retrospective review of 948 MRE studies performed between June 2009 and December 2012 at our institution. Clinical data (demographics, disease characteristics and therapy) were obtained from electronic patient records. Incidental findings were defined as unexpected lesions in or outside the small intestine, previously unknown or unsuspected at the time of referral and unrelated to IBD.

Results Of 948 MRE studies 445 patients had a diagnosis of IBD, 385 had CD, 54 had ulcerative colitis and 16 had IBD unclassified (IBDU).

Of 385 CD patients, 224 were female, mean age 36 (range 12-72) and median follow up of 4 years (range 0-39). Abnormalities were noted in 285 scans, 162 active non-stricturing, 109 active stricturing and 13-fibrostenosis. Within active groups were 29 fistulae and 12 abscesses in 33 patients. Incidental findings included colitis (10), gallstones (17), ovarian cyst (15), sacroileitis (1), renal cyst (10), hepatic cyst (10), splenic haemangioma (1), mesenteric abscess (1), adrenal nodule (2), uterine fibroid (4), chronic pancreatitis (1) and splenomegaly (2) associated with portal vein thrombosis in and varices.

70 studies were performed in UC or IBDU; mean age 34 (range 15-82) 39 were female. Small bowel thickening with signs of active inflammation were seen in $9 / 13$. Other findings included a fluid filled collection in the right ischio-anal fossa, pancreatic divisum, gallstones and liver, ovarian and Nabothian cysts, colitis in 6 and colonic polyps in 1 .

Indications for MRE in the non-IBD group (503 patients) included iron deficiency anaemia, abdominal pain, weight loss, diarrhoea, vomiting, abnormal colonoscopy or intra-abdominal abscess. Findings included small bowel thickening (4), sub-acute small bowel obstruction (2), small bowel malignancies (2), small bowel stricture (1) and small bowel intussusception (1). Incidental findings included ovarian, hepatic and renal cysts, adrenal adenoma, ascites, splenic and liver haemangioma, AAA, PUJ obstruction, liver metastases, gallstones, gallbladder polyp, pelvic abscess, uterine fibroids, large bowel stricture, diverticular disease, cirrhosis, lymphadenopathy, horseshoe kidney, atrophic pancreas and acute appendicitis.

Conclusion A small but significant proportion of patients have important incidental findings at MRE. MRE can add meaningfully to the investigation of SB pathology. A careful selection of patients can be achieved through a collaborative approach between radiologists and clinicians.

Disclosure of Interest None Declared.

\section{Poster presentation III}

\section{Colorectal/Anorectal}

\section{PTH-001 A NATIONAL SURVEY OF LOCAL HEREDITARY COLORECTAL CANCER SERVICES IN THE UK; A HIGHLY VARIABLE APPROACH?}

doi:10.1136/gutjnl-2013-304907.489

${ }^{1, *} \mathrm{~K}$ J Monahan, ${ }^{2} \mathrm{~S} \mathrm{~K}$ Clark on behalf of BSG Cancer Group. ${ }^{1}$ Family History of Bowel Cancer Clinic, West Middlesex University Hospital; 2Polyposis Registry, St Mark's Hospital, London, UK
Introduction The identification of inherited gastrointestinal disease provides an opportunity to prevent colorectal cancer. Heritable factors contribute about $35 \%$ of all colorectal cancer risk which has a significant impact on clinical activity in centres managing colorectal cancer. The British Society of Gastroenterology (BSG) and Association of Coloproctologists of Great Britain and Ireland (ACPGBI), released updated guidelines in 2010 for the management of patients with a family history of colorectal cancer. There is evidence that adherence to these guidelines is highly variable both for endoscopic screening and testing individuals for inherited conditions such as Lynch Syndrome and the Polyposis Syndromes. The aim of this survey was therefore to facilitate understanding of how services for patients with inherited colorectal cancer risk can be improved, and to raise awareness of this issue amongst clinicians.

Methods Following consultation within the BSG Cancer Group, UK Gastroenterologists, Colorectal Surgeons, Clinical and Medical Oncologists were invited to complete a short 10 point questionnaire. This was cascaded by email to 1,793 members of the Royal College of Radiologists (RCR), Association of Cancer Physicians (ACP), the BSG and ACPGBI. We sought their opinion and perception of local hereditary colorectal cancer services, also their adherence to and understanding of current national guidelines.

Results Three hundred and eighty-two members responded to the survey, an overall response rate of $21.3 \%$. Although $69 \%$ of respondents felt there was an adequate service for these patients, $64 \%$ also believed that another clinician was undertaking this work. There was no apparent patient pathway in 52\% of centres, and only $33 \%$ maintain a register of these patients. Patients rarely receive initial tumour block testing for Lynch Syndrome. When asked what they would like to augment the service they receive many respondents requested 'clear guidelines', 'pathways' and dedicated support networks. Many appeared to be unaware of the BSG/ACPGBI guidelines for the management of these patients.

Conclusion There was wide variability in practise and in pathways for hereditary colorectal cancer patients with a perception that they should be managed by another unspecified clinician. BSG/ACPGBI National Guidelines are not adhered to, therefore we recommend improved education, well defined pathways and audit in order to improve care of patients with hereditary colorectal cancer risk.

Disclosure of Interest None Declared.

\section{PTH-002 A SINGLE CENTRE AUDIT COMPARING COLONOSCOPY COMPLETION AND COMPLICATION RATES IN OLDER AND YOUNGER PATIENTS}

doi:10.1136/gutjnl-2013-304907.490

1.*'L Harrison, 'R Parish, 'R Shenderey. 'Gastroenterology, Airedale General Hospital, Keighley, UK

Introduction Colonoscopy is the gold standard test for large bowel assessment. Radiological techniques are available and have a high sensitivity and specificity for diagnosing colorectal cancer. ${ }^{1}$ As part of the Joint Advisory Committee (JAG) for endoscopy accreditation, units should achieve a caecal intubation rate of $>90 \%$. We audited our unit's performance.

Methods We audited colonoscopies carried out in 2011 at a District General Hospital. Endoscopy reports of 75 patients aged 80 and over and 75 patients under 75 years old were analysed.

Results 1635 colonoscopies were carried out by the unit in 2011 The pick-up rate for cancer was at least 3.5\% and for polyps was $23 \%$. Completion of colonoscopy in under 75 year olds was $97 \%$. Completion in over 80 year olds was $79 \%$. The unit's overall completion rate in 2011 was $90 \%$ (24\% terminal ileum, $65 \%$ caecum, $1 \%$ anastomosis). Colonoscopy was better tolerated in the younger group with discomfort suffered in $7 \%$ of patients compared to $11 \%$ of elderly patients (see table 1 ). The overall pick-up rate for cancer in both groups was 3\%. 